\title{
Evaluation of the removal reasons of totally implantable venous devices in children: a retrospective study
}

\author{
Sinan Kılıç, Tutku Soyer, İbrahim Karnak, Arbay Özden Çiftçi, Feridun Cahit Tanyel, \\ Mehmet Emin Şenocak \\ Department of Pediatric Surgery, Hacettepe University Faculty of Medicine, Ankara, Turkey. \\ E-mail: soyer.tutku@gmail.com \\ Received: 1 March 2016, Accepted: 31 May 2016
}

SUMMARY: Kılıç S, Soyer T, Karnak İ, Çiftçi AÖ, Tanyel FC, Şenocak ME. Evaluation of the removal reasons of totally implantable venous devices in children: a retrospective study. Turk J Pediatr 2016; 58: 187-194.

Totally implantable venous access devices (TIVADs) increase the quality of life in children with hematologic and oncologic diseases or organ failures. The aim of this study is to determine the reasons for port removal. The port catheters, implanted and removed in patients between January 2000 and June 2013 were evaluated retrospectively. The patients were divided into two groups, whose port catheters were removed due to completed therapy (completed therapy group, CTG) and whose port catheters were removed because of a port catheter-related complications (complication group, CG). In the CG, the patients whose port catheters are removed for infectious reasons are investigated for whether there is a relationship with age, gender, body mass index (BMI), height and weight at the time of port implantation and removal. In total, 242 patients who underwent port implantation and removal were included in the study. The male to female ratio was 1.32/1 and the mean age of the patients was $9.4 \pm 4.9$ years ( $0-24$ year). Patients were enrolled in CTG $(n=170,70.2 \%)$, and CG $(n=72,29 \%)$. There is a positive correlation between BMI and infections $(\mathrm{p}<0.05)$. In the CG, patients under steroid treatment had higher incidence of non-infectious causes than infectious causes $(\mathrm{p}<0.05)$. Oppositely, non infectious complications were higher in steroid free patients $(\mathrm{p}<0.05)$. There was no catheter related mortality in the entire study group.

The hematological malignancies and solid tumors are the most common underlying primary disease in patients with port removal because of complications. Infectious complications are most common cause of port removal in children and despite other microorganism, fungi should be considered as a cause of catheter related infections.

Key words: totally implantable venous catheter, children, cancer, infection, port.

Totally implantable venous access devices (TIVADs) improve the quality of life and healthcare services provided to patients with cancer because they allow long term intravenous (IV) access for chemotherapy, antibiotherapy, blood transfusion and so on. In addition, they have an advantage for venous blood sampling without having to puncture the vein repetitively. Their usage has been accepted widely, especially since 1980s, and they are preferred over central venous catheters in oncologic patients receiving chemotherapy.
Due to easy access for IV line, port catheters improve the quality of treatment and patient adaptation in oncologic patients ${ }^{1-3}$. However, like the other central venous routes, port catheters have some early and late term complications. Some of these complications may sometimes become more severe than the primary disease itself. The complications related to procedure are pneumothorax, hemothorax, air embolism, arterial puncture, arrhythmia, pericardial tamponade and brachial plexus injury ${ }^{4-6}$. The short term complications after the procedure include hemoptysis, hematoma 
in the port implantation site, dehiscence of the incision ${ }^{7,8}$; whereas late complications include catheter related infections, thrombosis of the catheter, tear and transection of the catheter, 'pinch-off' syndrome, relocation of the catheter tip, embolization due to catheter, extravasations of the given medications, slipping of the catheter, erosion and/or perforation of superior vena cava ${ }^{9-14}$. However, these short and late complications may be observed in both terms.

The aim of this study is to determine the reasons for port removal and to compare the characteristics of patients whose ports were removed due to completed therapy and whose ports were removed due to port catheter related complications. Therefore, a retrospective study was performed to evaluate the causes of port removal in children.

\section{Material and Methods}

The patients who underwent port implantation and removal at the Department of Pediatric Surgery between January 2000 and June 2013 were evaluated retrospectively. The patients were evaluated for age, gender, primary diagnosis, height, weight and body mass index (BMI) of the patient at the time of implantation and removal of port catheter, the access vein of the port, port size, complications during implantation and follow-up, usage of steroids, reasons for port removal, port infections and their characteristics (catheter culture and antibiogram results) and survival rates. The data of the patients are obtained from the patient files and from the online hospital documentation systems.

The insertion criteria for port catheters in our Children's Hospital include long term intravenous access for chemotherapy and blood infusions. According to chemotherapy protocols, pediatric oncology and hematology patients offered port insertion and port catheters were inserted surgically after obtaining consent of the parents. Cuffed-tunnel catheters were used for short term intravenous nutrition and hemodialysis. The patients, whose port catheters were implanted by the interventional radiology unit or other institutes, were not included in the study.

Surgical intervention for port insertion and removal: Under general anesthesia, port catheters were inserted with cut down for external jugular veins and purse-string technique for internal jugular veins. Ports were inserted subcutaneously with small incisions on chest wall and catheters were implanted to ports via guided subcutaneous tunnel. For port removal, ports and catheters were freed form surrounding pseudo-capsule and removed from previous incision.

Definition of port infections and port thrombosis: The wound of the port site and dressing of ports are evaluated daily in oncology and hematology patients. Care and dressing of port catheters were managed by the Guidelines for prevention of intravascular catheter-related infections ${ }^{15}$. Wound infection of patients was assessed according to criteria for Guideline for Prevention of Surgical Site Infection adopted by Center for Disease Control Prevention $(\mathrm{CDC})^{16}$. Definition of port site infections were monitored during each dressing and tenderness in the port site, fever without obvious source and other manifestations suggesting local and/or blood steam infections are considered for port infection ${ }^{15}$. Wound swabs and blood steam cultures were obtained for definite

Table I. Demographic Characteristics of the Patients and Their Distribution Across the Groups

\begin{tabular}{lcccc}
\hline & & CTG & CG & p \\
\hline Age (years) & & $10.2 \pm 4.9$ & $7.7 \pm 4.4$ & $>0.05$ \\
Gender (M/F) & & $1.32 / 1$ & $1.15 / 1$ & $>0.05$ \\
Weight $(\mathrm{kg})$ & During implantation & $17.9 \pm 1.2$ & $14.1 \pm 9.4$ & $>0.05$ \\
& During removal & $27 \pm 1.6$ & $16 \pm 9.3$ & $>0.05$ \\
Height $(\mathrm{cm})$ & During implantation & $100.8 \pm 29.3$ & $92 \pm 24$ & $>0.05$ \\
& During removal & $110 \pm 29$ & $96 \pm 23$ & $>0.05$ \\
Steroid usage & & $58(34.1 \%)$ & $35(49.3 \%)$ & $>0.05$ \\
\hline M: male; F: female; CTG: completed therapy group; CG: complication group
\end{tabular}

M: male; F: female; CTG: completed therapy group; CG: complication group 
diagnosis. Wound infections are treated with appropriate antimicrobials, debridement and abscess drainage. Dehiscence of port catheters due to wound infection and systemic infection findings according to catheter infections were considered for port removal.

Occluded ports were evaluated for thrombosis by Doppler USG and anticoagulant treatment was used for these patients. Patients with occluded catheters due to massive thrombosis and unresponsive to anticoagulant treatment were considered for port removal.

The patients were divided into two groups, whose port catheters were removed due to completed therapy (completed therapy group, CTG) and whose port catheters were removed because of a port catheter related complications (complication group, CG).

The study protocol was approved by the Institutional Non-interventional Clinical Studies Ethical Committee (Date: 12.07.13, Registration No: 16999557-775).

\section{Statistical Analysis}

The data of the patients were compared with SPSS 16.0 and descriptive studies were obtained. The data of patients regarding CTG and CG were compared by using Chisquare test. The $\mathrm{p}$ values less than 0.05 were considered as significant.

\section{Results}

A total of 242 patients who underwent port implantation and removal between January 2000 and June 2013 were included in the study. Among 242 patients $132(54.5 \%)$ of children were male, and 110 (45.5\%) were female. The male to female ratio was $1.32 / 1$. The mean age of the patients was $9.4 \pm 4.9$ years $(0-24$ year). Patients were enrolled in CTG $(n=170$, $70.2 \%)$, and CG $(n=72,29.8 \%)$.

The demographic characteristics and steroid use are summarized in Table I; demographic features showed no significant difference in both groups $(p>0.05)$.

Ninety-three patients (38.6\%) received steroid treatment during follow-up. The rest of the patients 148 (61.4\%) did not receive steroid during follow-up. The incidence of steroid treatment was similar in groups $(p>0.05)$ (Table I).

The patients are divided into four groups according to their primary disease including: hematological malignancy $(\mathrm{n}=77,31.8 \%)$, solid tumors $(\mathrm{n}=94,38.8 \%)$, other malignancies $(\mathrm{n}=57,23.6 \%)$, and non-tumoral indications $(n=14,5.8 \%)$ for port implantation. The distribution of patients were similar in four primary diseases $(\mathrm{p}>0.05)$.

Among hematological malignancies, acute lymphoblastic leukemia (ALL) was the most common primary disease for port implantation $(n=41,16.9 \%)$. There was no significance between groups for the distribution of hematological malignancy subtypes ( $p>0.05)$.

Neuroblastoma ( $\mathrm{n}=40,16.5 \%)$, Wilms' tumor $(n=20,8.3 \%)$, retinoblastoma $(n=22,9.1 \%)$ and primitive neuroectodermal tumor (PNET) $(n=8,3.3 \%)$ were the most common solid tumors in groups with similar incidences in CTG and CG ( $p>0.05)$.

Non-tumoral indications for port catheter implantation include, chronic diarrhea/

Table II. The Distribution of Access Vein

\begin{tabular}{|c|c|c|c|c|c|c|c|}
\hline \multirow{2}{*}{ Site of access } & \multicolumn{2}{|c|}{ CTG } & \multicolumn{2}{|c|}{ CG } & \multicolumn{2}{|c|}{ Total } & \multirow{2}{*}{$\mathrm{p}$} \\
\hline & $\mathrm{n}$ & $\%$ & $\mathrm{n}$ & $(\%)$ & $\mathrm{n}$ & $(\%)$ & \\
\hline R EJ & 117 & $69.2 \%$ & 50 & $69.4 \%$ & 167 & $69.3 \%$ & $>0.05$ \\
\hline R IJ & 41 & $24.3 \%$ & 17 & $23.6 \%$ & 58 & $24.1 \%$ & $>0.05$ \\
\hline L EJ & 7 & $4.1 \%$ & 4 & $5.6 \%$ & 11 & $4.6 \%$ & $>0.05$ \\
\hline L IJ & 3 & $1.8 \%$ & 0 & & 3 & $1.2 \%$ & $>0.05$ \\
\hline Great saphenous vein & 0 & & 1 & $1.4 \%$ & 1 & $1.4 \%$ & $>0.05$ \\
\hline Femoral vein & 1 & $0.6 \%$ & 0 & & 1 & $0.4 \%$ & $>0.05$ \\
\hline Total & 169 & & 72 & & 241 & & $>0.05$ \\
\hline
\end{tabular}

R: right; L: left; EJ: external jugular; IJ: internal jugular; CTG: completed therapy group; CG: complication group 
Table III. The Causes of Port Removal in the Complication Group

\begin{tabular}{lcc}
\hline & $\mathrm{N}$ & $\%$ \\
\hline Infection & 37 & 51.4 \\
Dehiscence of incision site & 13 & 18.1 \\
Extravasation & 7 & 9.7 \\
Mechanical problems & 7 & 9.7 \\
Thrombosis & 6 & 8.3 \\
Exchange of the catheter with dialysis catheters & 2 & 2.8 \\
\hline
\end{tabular}

malnutrition ( $\mathrm{n}=5,21 \%)$, short gut syndrome $(n=2,0.8 \%)$, prematurity $(n=1,0.4 \%)$, immune deficiencies $(n=1,0.4 \%)$, Beckwith-Wiedemann syndrome $(n=1,0.4 \%)$, hemophagocytic syndrome $(\mathrm{n}=1,0.4 \%)$, Joubert syndrome $(\mathrm{n}=1,0.4 \%)$, hemolytic uremic syndrome (HUS) $(n=1,0.4 \%)$. This group of patients had TIVADs after several other complicated catheter insertions. Also, there was no significant difference between groups for distribution of non-tumoral indications $(\mathrm{p}>0.05)$.

The diameters of port catheter lumens were 4.5 Fr, 5 Fr, 6 Fr, 6.5 Fr and more than $7 \mathrm{Fr}$ (up to $9 \mathrm{Fr}$ ) catheters were used in adolescent patients. One hundred and twenty-four of cases $(51.2 \%)$ received $6.5 \mathrm{Fr}$ catheter, $86(35 \%)$ of cases received $4.5 \mathrm{Fr}, 12$ of $(5 \%)$ cases received $7.6 \mathrm{Fr}$, and $8(3 \%)$ of them received $8.5 \mathrm{Fr}$ sized port catheters. There is no significance between groups for distribution of port catheter lumen diameters $(\mathrm{p}>0.05)$.

When we analyzed the access vein for port catheters; external jugular, internal jugular, great saphenous and the femoral veins were the sites for port catheter access. While the great saphenous vein and femoral vein are used in one case each, jugular veins were the most common sites for vascular access. In 14 of cases
$(5.8 \%)$, left side of the neck is preferred due to thrombus, mass or infection in the right side. Left external jugular vein was chosen in 11 of such cases, while 3 of them were performed from left internal jugular vein.

When site of port accesses were analyzed CTG and CG did not show significant difference (Table II).

There were no complications in $97.5 \%$ of the cases $(n=236)$ during the port implantation procedure. The displacement of catheter into right ventricle $(n=3,1.2 \%)$, hematoma $(n=2$, $0.8 \%$ ) and disconnection of port reservoir and port catheter $(n=1,0.3 \%)$ were the intra-operative complications. These early complications during the procedure are managed with hemostasis, debridement and catheter revision.

The causes for port removal in the CG are shown in Table III. Infectious reasons were the most common complications (51.4\%).

When we investigated the amount of time with port catheters, the mean duration of a port catheter was $864 \pm 556$ days (7-3,696 days). In CTG, the mean duration of port catheter was 1,043 days (24-2,917 days), while it was 443 days (7-3,696 days) in CG. The mean duration

Table IV. The Interventions During Follow-Up of Port Catheters

\begin{tabular}{lcc}
\hline & $\begin{array}{c}\text { Completed therapy group } \\
(\mathrm{n})\end{array}$ & $\begin{array}{c}\text { Complication group } \\
(\mathrm{n})\end{array}$ \\
\hline No intervention & 164 & 56 \\
Anticoagulation & 3 & 4 \\
Debridment & 0 & $6^{*}$ \\
Drainage for abscess & 1 & $5^{*}$ \\
Pulling up of port catheter & 1 & 1 \\
Hemostasis & 1 & 0 \\
\hline
\end{tabular}

*Despite the successful intervention, the port catheter needed to be removed. 
Table V. Infectious Agents Reproduced in the Catheter Blood Culture

\begin{tabular}{lccc}
\hline & $\begin{array}{c}\text { Completed therapy } \\
\text { group } \\
(\mathrm{n})\end{array}$ & $\begin{array}{c}\text { Complication group } \\
(\mathrm{n})\end{array}$ & Total \\
\hline No organism & 166 & 33 & 199 \\
Staphylococcus aureus & 0 & 11 & 11 \\
Candida & 1 & 13 & 14 \\
Staphylococcus epidermidis & 1 & 6 & 7 \\
Candida + Staphylococcus & 0 & 2 & 2 \\
MRSA (Methicillin-resistant & 2 & 1 & 3 \\
Staphylococcus aureus) & 0 & 3 & 3 \\
Klebsiella & 0 & 2 & 2 \\
Pseudomonas & 0 & 1 & 1 \\
Acinetobacter baumani & 0 & & \\
\hline
\end{tabular}

Table VI. The Correlation Between Causes of Port Removal and Primary Disease

\begin{tabular}{|c|c|c|c|c|c|}
\hline & \multicolumn{2}{|c|}{ Infection } & \multicolumn{2}{|c|}{ Non-infectious reasons } & \multirow[b]{2}{*}{$\mathrm{p}$} \\
\hline & $\mathrm{n}$ & $(\%)$ & $\mathrm{n}$ & $(\%)$ & \\
\hline Hematological malignancies & 14 & 37.8 & 11 & 31.4 & $>0.05$ \\
\hline Solid tumors & 14 & 37.8 & 12 & 34.3 & $>0.05$ \\
\hline Other malignancies & 4 & 10.8 & 9 & 25.7 & $>0.05$ \\
\hline Non-tumoral indications & 5 & 13.5 & 3 & 8.6 & $>0.05$ \\
\hline
\end{tabular}

of port catheter was 431 days (63-1449 days) because of infectious complications and 455 days (7-3,696 days) for other complications. The time duration of port usage was similar between patients whose ports were removed for infectious complications and other $(\mathrm{p}>0.05)$.

During the follow-up, $9.1 \%$ of patients faced complications. These complications were managed with different treatment interventions (Table IV). In 199 (82.2\%) of patients, blood cultures obtained from catheters were negative for organisms. Fungi were the most common microorganism and followed by $S$. aureus and $S$. epidermidis in blood cultures. The antimicrobial characteristics of catheter-related complications were listed in Table V.

When the causes for port removal and primary diseases were correlated in the CG, patients with hematological malignancies and solid tumors had higher incidence of infectious causes ( $p>0.05$; Table VI). But there was no significance between patients with hematological malignancies and solid tumors of infectious causes. In the CG, the patients whose port catheters were removed for infectious reasons were investigated for whether there is a relationship with age, gender, BMI, height and weight at the time of port implantation and removal. There is a positive correlation between BMI and infections $(p<0.05)$ Children with higher BMI had higher incidence of infectious complications. In the CG, patients under steroid treatment had higher incidence of non-infectious causes $(n=21,60 \%)$ than infectious causes $(n=14,40 \%)(p<0.05)$ Oppositely, non infectious complications were higher in steroid free patients $(n=22,62.8 \%$, $\mathrm{p}<0.05)$.

\section{Discussion}

Difficulties in venous blood sampling or venous line access decrease the compatibility of the patient to treatment in pediatric age group. Port catheters improve the quality of life and healthcare services provided to patients with cancer, since they allow long term IV access for chemotherapy, antibiotherapy and blood transfusion ${ }^{17}$. In addition, they have an advantage for venous blood sampling without having to puncture the vein. In recent years, usage of port catheters in oncologic patients has been widely accepted, and it 
is preferred to other central venous access routes $^{18}$. In our institution, almost all patients who need long term chemotherapy undergo port catheter implantation. Apart from patients with cancer, children who require fluid therapy or blood products for chronic diarrhea, short gut syndrome, hemophagocytic syndrome, and hemolytic uremic syndrome may also undergo port catheter implantation.

There are a number of studies related to port catheter complications. However, there is no study regarding the characteristic of patients who have undergone port catheter removal or there is no such study which deals with the reasons for port removal in the complicated patients. Therefore this retrospective study is the first to analyze the causes of port removal in children.

In our study, we found that $29.8 \%$ of patients underwent port removal because of port catheter related complications. Hollyoak et al. ${ }^{18}$ reported that $75 \%$ of port catheters is removed due to completed therapy in adults ${ }^{19}$. The incidence of port removal for infectious complications accounts for $31.4 \%$ of all port removals in children ${ }^{20}$. These results were compatible with our findings and we suggest that in about 1 of 3 cases, port catheters are removed before completing the therapy due to port related complications in children.

In another study, patients who underwent port removal were below 2 years of age $(41.3 \%)^{21}$. Since we only include patients with removed ports, patients in our study were older $(>9$ year of age) than other patients reported in previous studies, however, demographic features of patients did not show significant difference in CTG and CG. These findings are similar to that of previous studies ${ }^{22,23}$.

In our patient population, solid tumors $(38.8 \%)$ and hematological malignancies $(31.8 \%)$ were the most common primary disease in children with port removal. Yazıc1 et al. ${ }^{20}$ reported that Non-Hodgin lymphoma is the most common primary malignancy in patients with infectious complications $^{21}$. We found that ALL is the most common malignancy in children and infectious complications were more common in hematologic malignancies and solid tumors.

The intraoperative complication rate was limited to $2.5 \%$ of all procedures in our patients and can be easily overcome during the implantation. Right jugular vein is preferable in all patients but left jugular veins may be used for port access. Also external veins were more preferred sites because of fewer complications. Tretola et al. ${ }^{23}$ suggest that jugular veins showed less complications (thrombosis and infections) when compared to subclavian veins. The lumen size of catheter and duration of catheters were not found as a cause of complications in hemodialysis catheters ${ }^{24}$. Also, we could not find any correlation between port size, duration of port access and complications.

When we investigate the patients who underwent port removal because of complications, the most common factor for port removal was infections (51.4\%). We found that patients with hematological malignancies or solid tumors were more prone to develop port infections. On the other hand, other underlying diseases showed non-infections complications. Since, patients with hematologic disease show altered immunity and develop severe neutropenia, infectious complications are more likely to be detected. In a previous study, the incidence of port infection was $14 \%$ and infection rate was reported to be $1.92 / 1000$ catheter days ${ }^{21}$. Port infections can be classified as sepsis, port reservoir site infection and catheter tunnel infections. Although port reservoir site infections were less than sepsis, incidence of $26.3 \%$ was reported by Yazıc1 et al. ${ }^{20}$. However, only $5 \%$ of our patients developed reservoir site infections and no catheter tunnel infection was detected in our patients.

In previous studies, the most common infectious agent is reported as S. epidermidis in port catheter related infections ${ }^{15,25}$. Also, coagulase negative staphlococci (S. epidermidis, S. hominis, S. saprophyticus) were reported as the most common microbial organisms ${ }^{22}$. In our study, different from other, we found that fungi were the most common and were followed by $S$. aureus and S. epidermidis. The reasons for higher incidence of fungi are thought to be the long duration of catheters inside the body and increased usage of total parenteral nutrition through port catheters ${ }^{15,26}$. Especially in immune-compromised patients and patients under extensive antibiotherapy regimens, the frequency of Candida (C. albicans, C. parapsilosis) and other fungi (Fusarium, 
Malassezia furfur, Rhodotorula, Trichosporon) significantly increases ${ }^{26-27}$. We suggest that longer duration of port catheters with an underlying hematological diseases are the possible causes of higher fungi infection in port cultures.

In this study, we could not find any correlation with infectious complications and age, gender, height and weight at the time of port implantation and removal in the CG. However, there is a positive relation between infections and higher BMI.

Although higher incidence of infectious complications were expected in patients under long term steroid treatment because of altered immune system and neutropenia, we found that patients with steroid treatment were more prone to develop non-infectious complications. Therefore, we suggest that the reasons of the higher incidence of non-infectious causes in patients under steroid therapy might be conservation of port catheter and reservoir under a thinner skin and subcutaneous adipose tissue and the negative effects of steroids on wound healing.

The catheter related mortality has been reported between $2.8 \%-3.5 \% 28$. It has been suggested that recurrent disease and neutropenia may contribute to catheter-related mortality ${ }^{21}$. In our study, there was no catheter-related mortality. In the follow-up, $16 \%$ of the patients did not receive another port catheter and their treatment continued with another central venous route. However, $74 \%$ of the patients underwent new port catheter implantation.

In conclusion, one third of the port catheters in the pediatric population are removed before completing the treatment due to various complications. The hematological malignancies and solid tumors are the most common underlying primary disease in patients with port removal because of complications. Infectious complications are the most common cause of port removal in children and despite form, other microorganism fungi should be considered as a cause of catheter-related infections. It should be kept in mind that patients with higher BMI are more prone to infectious complications.

\section{REFERENCES}

1. Funaki B. Central venous access: a primer for the diagnostic radiologist. AJR Am J Roentgenol 2002; 179: 309-318.

2. Biffi R, Toro A, Pozzi S, Di Carlo I. Totally implantable vascular access devices 30 years after the first procedure. What has changed and what is still unsolved? Support Care Cancer 2014; 22: 1705-1714

3. Coppa GF, Gouge TH, Hofstetter SR. Air embolism: a lethal but preventable complication of subclavian vein catheterization. JPEN J Parenter Enteral Nutr 1981; 5: 166-168.

4. Ezaru CS, Mangione MP, Oravitz TM, Ibinson JW, Bjerke RJ. Eliminating arterial injury during central venous catheterization using manometry. Anesth Analg 2009; 109: 130-134.

5. Faraj W, Zaghal A, El-Beyrouthy O, Kutoubi A. Complete catheter disconnection and migration of an implantable venous access device: the disconnected cap sign. Ann Vasc Surg 2010; 24: 692.e11-e15.

6. Burns S, Herbison GJ. Spinal accessory nerve injury as a complication of internal jugular vein cannulation. Ann Intern Med 1996; 125: 700.

7. Wiegand UK, LeJeune D, Boguschewski F, et al. Pocket hematoma after pacemaker or implantable cardioverter defibrillator surgery: influence of patient morbidity, operation strategy, and perioperative antiplatelet/ anticoagulation therapy. Chest 2004; 126: 1177-1186.

8. Zawacki WJ, Walker TG, DeVasher E, et al. Wound dehiscence or failure to heal following venous access port placement in patients receiving bevacizumab therapy. J Vasc Interv Radiol 2009; 20: 624-627.

9. Flynn PM, Shenep JL, Stokes DC, Barrett FF. In situ management of confirmed central venous catheterrelated bacteremia. Pediatr Infect Dis J 1987; 6: 729734.

10. de Jonge RC, Polderman KH, Gemke RJ. Central venous catheter use in the pediatric patient: mechanical and infectious complications. Pediatr Crit Care Med 2005; 6: $329-339$

11. Lokich JJ, Bothe A Jr, Benotti P, Moore C. Complications and management of implanted venous access catheters. J Clin Oncol 1985; 3: 710-717.

12. Kurul S, Saip P, Aydin T. Totally implantable venousaccess ports: local problems and extravasation injury. Lancet Oncol 2002; 3: 684-692.

13. Biffi R, de Braud F, Orsi F, et al. Totally implantable central venous access ports for long-term chemotherapy. A prospective study analyzing complications and costs of 333 devices with a minimum follow-up of 180 days. Ann Oncol 1998; 9: 767-773.

14. Jueckstock JK, Mylonas I, Strobl B, Willgeroth F, Sommer HL, Friese K. Paravasation with cyclophosphamide case report of tissue necrosis in a patient with primary breast cancer. Onkologie 2007; 30: 452-454.

15. O'Grady NP, Alexander M, Burns LA, et al. Guidelines for the prevention of intravascular catheter-related infections. Am J Infect Control 2011; 39: (4 Suppl 1) S1-S34. 
16. Mangram AJ, Horan TC, Pearson ML, Silver LC, Jarvis WR, Guideline for prevention of surgical site infection. 1999 Hospital Infection Control Practices Advisory Committee. Infect Control Hosp Epidemiol 1999; 20: 250-278.

17. Filippou DK, Tsikkinis C, Filippou GK, Nissiotis A, Rizos S. Rupture of totally implantable central venous access devices (Intraports) in patients with cancer: report of four cases. World J Surg Oncol 2004; 2: 36.

18. Jost K, Leithauser M, Grosse-Thie C, et al. Perforation of the superior vena cava - a rare complication of central venous catheters. Onkologie 2008; 31: 262-264.

19. McMahon C, Smith J, Khair K, Liesner R, Hann IM. Smith OP. Central venous access devices in children with congenital coagulation disorders: complications and long-term outcome. Br J Haematol 2000; 110: 461-468.

20. Yazıcı N, Akyüz C, Yalçın B, Varan A, Kutluk T, Büyükpamukçu M. Infectious complications and conservative treatment of totally implantable venous access devices in children with cancer Turk J Pediatr 2013; 55: 164-171.

21. Ingram J, Weitzman S, Greenberg ML, Parkin P, Filler R. Complications of indwelling venous access lines in the pediatric hematology patient: a prospective comparison of external venous catheters and subcutaneous ports. Am J Pediatr Hematol Oncol 1991; 13: 130-136.
22. Hollyoak MA, Ong TH, Leditschke JF. Critical appraisal of surgical venous access in children. Pediatr Surg Int 1997; 12: 177-182.

23. Trerotola SO, Kuhn-Fulton J, Johnson MS, Shah H, Ambrosius WT, Kneebone PH. Tunneled infusion catheters: increased incidence of symptomatic venous thrombosis after subclavian versus internal jugular venous access. Radiology 2000; 217: 89-93.

24. Özbudak E, Yavuz Ș, Akgül A ve ark. Kalıcı hemodiyaliz kateterleri: Ne kadar kalıcı? Türk Göğüs Kalp Damar Cerrahisi Dergisi 2013; 21: 646-653.

25. Chauhan A, Lebeaux D, Ghigo JM, Beloin C. Full and broad-spectrum in vivo eradication of catheterassociated biofilms using gentamicin-EDTA antibiotic lock therapy. Antimicrob Agents Chemother 2012; 56: 6310-6138.

26. National Nosocomial Infections Surveillance (NNIS) System Report, Data Summary from January 1990May 1999, Issued June 1999. A report from the NNIS System. Am J Infect Control 1999; 27: 520-532.

27. Schaberg DR, Culver DH, Gaynes RP. Major trends in the microbial etiology of nosocomial infection. Am J Med 1991; 91: 72S-75S

28. Abbas AA, Fryer CJ, Paltiel C, et al. Factors influencing central line infections in children with acute lymphoblastic leukemia results of a single institutional study. Pediatr Blood Cancer 2004; 42: 325-331. 